\title{
LINE TRANSECTS, COVARIANCE FUNCTIONS AND SET CONVERGENCE
}

\author{
A. J. CABO, ${ }^{*}$ CWI, Amsterdam \\ A. J. BADDELEY,** University of Western Australia and University of Leiden
}

\begin{abstract}
We define the 'linear scan transform' $G$ of a set in $\mathbb{R}^{d}$ using information observable on its one-dimensional linear transects. This transform determines the set covariance function, interpoint distance distribution, and (for convex sets) the chord length distribution. Many basic integral-geometric formulae used in stereology can be expressed as identities for $G$. We modify a construction of Waksman (1987) to construct a metric $\eta$ for 'regular' subsets of $\mathbb{R}^{d}$ defined as the $L^{1}$ distance between their linear scan transforms. For convex sets only, $\eta$ is topologically equivalent to the Hausdorff metric. The set covariance function (of a generally non-convex set) depends continuously on its set argument, with respect to $\eta$ and the uniform metric on covariance functions.
\end{abstract}

LINEAR SCAN TRANSFORM; CHORD LENGTH DISTRIBUTION

AMS 1991 SUBJECT CLASSIFICATION: PRIMARY 60D05

\section{Introduction}

This paper studies the determination of a set $A \subset \mathbb{R}^{d}$ from information on one-dimensional linear transects $A \cap l$. Three issues are discussed:

(a) characterisation: whether a set $A \subset \mathbb{R}^{d}$ is completely determined by the values of an associated transform (the Radon transform, chord length distribution, interpoint distance distribution, or set covariance function);

(b) stereology: whether geometrical parameters of $A \subset \mathbb{R}^{d}$ can be statistically estimated from randomly-sampled values of the transforms;

(c) approximation: whether good deterministic or stochastic approximation of transforms ensures closeness of the corresponding sets.

Although such problems have received much attention, it is common for (a)-(c) to be considered separately. In this paper we introduce a transform that is serviceable in all three contexts.

Following is a brief summary of known results. A bounded open set is essentially uniquely determined by its Radon transform. When the available information is

Received 8 July 1993; revision received 1 February 1995.

* Postal address: Lindenheuvel 16, 1217 JX Hilversum, The Netherlands.

** Postal address: Department of Mathematics, University of Western Australia, Nedlands, WA 6009, Australia.

The original version of this paper was presented at the International Workshop on Stochastic Geometry, Stereology and Image Analysis held at the Universidad Internacional Menendez Pelayo, Valencia, Spain, on 21-24 September 1993. 
merely the probability distribution of chord lengths, characterisation is not guaranteed. Mallows and Clark (1970) provided a counterexample of two non-congruent convex polygons with the same chord length distribution.

For convex sets there are well-known integral geometric relationships through which the chord length distribution is uniquely determined by, and uniquely determines, the interpoint distance distribution, which in turn is determined by the set covariance function (Matérn (1985)). For stereological purposes ((b) above) there is an identity of Crofton (1885) concerning the moments of chord lengths which was generalised to non-convex sets by Miles (1979) and Jensen and Gundersen (1985), see also Goodey and Weil (1992).

There is much recent interest in the characterisation of a set from its set covariance function. Nagel (1991), (1993) showed that a convex plane polygon is uniquely determined by its set covariance. Lešanovský and Rataj (1990) constructed a counterexample of two distinct non-convex polygons with identical covariance functions. For a more restricted class of 'generic' polygons, not necessarily convex, which excludes the latter counterexample, the covariance function uniquely characterises the polygon, and there is a reconstruction procedure due to Schmitt (1993).

Suppose the intersection $A \cap l$ of a line $l$ with a set $A \subset \mathbb{R}^{d}$ is a finite union of compact intervals, with ordered endpoints $x_{1}, x_{2}, \cdots, x_{2 n}$. Miles (1983) defined the $k$-linc

$$
\left[\sigma(A \cap l)^{k}\right]=\sum_{i=1}^{2 n-1} \sum_{j=i+1}^{2 n}(-1)^{i+j+1}\left(x_{j}-x_{i}\right)^{k}, \quad \text { for } k \geqq 1
$$

and proved several integral-geometric identities for it. Waksman (1987) introduced the glance function of $A$,

$$
H_{A \cap l}(t)=\sum_{i=1}^{2 n-1} \sum_{j=i+1}^{2 n}(-1)^{i+j+1} 1\left\{x_{j}-x_{i} \leqq t\right\}, \quad \text { for } t \geqq 0
$$

which has an obvious resemblance to the $k$-linc. Waksman used this function to construct a metric on a class of open subsets of the plane, with applications to the approximation problem (c).

The new geometric transform introduced in this paper is a minor modification of Waksman's glance function but turns out to be extremely natural, arising as minus the derivative of the one-dimensional set covariance of a linear transect. Many integral geometric identities can be rewritten in terms of the linear scan transform.

The plan of the paper is as follows. Section 1 provides necessary background. The linear scan transform is defined in Section 2. Section 3 explores properties of the transform including relations with the $k$-linc, volume and other quantities. A generalisation of Crofton's formula to non-convex sets is stated. Stereological estimators can be constructed in terms of the linear scan transform.

In Section 4 we pursue the approximation problem by constructing a metric $\eta$ on 
'regular' subsets of $\mathbb{R}^{d}$, defined as the $L^{1}$-distance between their linear scan transforms. In the convex case, $\eta$ is the $L^{1}$ distance between Radon transforms. One of our main results (Theorem 4.7) is that for convex sets (but not in general) $\eta$ is topologically equivalent to the Hausdorff metric.

In the final section we obtain analytic properties of the set covariance function $C_{A}$ of subsets $A \subset \mathbb{R}^{d}$. For the class $\mathscr{V}$ of 'regular' sets equipped with the metric $\eta$, we show (Corollary 5.6) that the map $A \mapsto C_{A}$ is continuous from $\mathscr{V}$ into the space of bounded real functions on $\mathbb{R}^{d}$ with the uniform (supremum) metric, and (Lemma 5.1) that Lebesgue volume is a Lipschitz continuous function with constant 1.

\section{Preliminaries}

(a) Notation. The context of this paper is Euclidean space $\mathbb{R}^{d}$ with norm $\|\cdot\|$. Throughout $\lambda_{d}$ or $\lambda$ denotes $d$-dimensional Lebesgue measure on the Borel $\sigma$-field $\mathscr{B}=\mathscr{B}\left(\mathbb{R}^{d}\right)$, and $\mathscr{H}^{d-1}$ is $(d-1)$-dimensional Hausdorff measure (Simon (1983), p. 6). Write $\bar{S}, S^{\circ}$ for topological closure and interior of $S$ respectively. The open ball in $\mathbb{R}^{d}$ with radius $r$ and centre $x$ is denoted by $B(x, r)$ and

$$
\kappa_{d}=\frac{2 \pi^{d / 2}}{d \Gamma(d / 2)}=\lambda(B(0,1))
$$

Denote by $\mathscr{L}$ the class of all one-dimensional lines in $\mathbb{R}^{d}$, and by $\mu$ the normalised invariant measure on $\mathscr{L}$ (see Santaló (1976), pp. 28, 200). For a Borel set $S \subset \mathbb{R}^{d}$, define $[S]=\{l \in \mathscr{L}: l \cap S \neq \varnothing\}$, the set of lines that intersect $S$.

(b) Covariance functions

Definition 1.1. The (set) covariance function of a bounded Borel set $S \subset \mathbb{R}^{d}$ is the function $C_{S}: \mathbb{R}^{d} \rightarrow \mathbb{R}_{+}$defined by .

$$
C_{S}(y):=\lambda\left(S \cap T_{y} S\right), \quad y \in \mathbb{R}^{d} .
$$

where we write

$$
T_{y} S=S-y=\{s-y: s \in S\}
$$

for the translation of $S$ by a vector $y \in \mathbb{R}^{d}$.

Observe that the covariance function is measurable as a function of $y ;$ the function $g(u, w)=1_{s}(u) 1_{s}(u+w)$ is clearly measurable and integrable on $\mathbb{R}^{2 d}$ so Fubini's theorem guarantees measurability of $C_{S}($.$) .$

The following properties are immediate consequences of Definition 1.1:

Lemma 1.2 .

(a) $C_{S}(0)=\lambda(S)$.

(b) $C_{S}$ has compact support: $C_{S}(y)=0$, for all $y \in \mathbb{R}^{d}$ with $\|y\| \geqq \operatorname{diam}(S)$. 
(c) $C_{S}$ is symmetric: $C_{S}(y)=C_{S}(-y)$, for all $y \in \mathbb{R}^{d}$.

Lemma 1.3 (Borel's overlap formula). For any integrable $f: \mathbb{R}_{+} \rightarrow \mathbb{R}$

$$
\int_{S} \int_{S} f(\|u-v\|) d u d v=\int_{\mathbb{R}^{d}} f(\|w\|) C_{S}(w) d w .
$$

In particular

$$
\int_{\mathbb{R}^{d}} C_{S}(y) d \lambda(y)=\lambda(S)^{2} .
$$

Equation (1.1) is proved by a change of variables $w=u-v$, see Borel (1947). The second formula is the special case $f \equiv 1$.

Clearly (1.1) connects the covariance function of $S$ with the distribution function of the distance between two independent uniformly distributed points in $S$ (see for example Nagel (1993)).

The following well-known result is a consequence of the Blaschke-Petkantschin formula (see Santaló (1976), Equation (4.2) p. 46 and Equation (12.23) p. 201; Miles (1985)).

Lemma 1.4. For integrable $f: \mathbb{R}_{+} \rightarrow \mathbb{R}$

$$
\int_{S} \int_{S} f(\|u-v\|) d u d v=\int_{\mathscr{L}} \int_{l \cap S} \int_{l \cap S}|s-t|^{d-1} f(|s-t|) d s d t d \mu(l) .
$$

(c) Regular sets. A set $S$ is called regular closed if $S=\overline{S^{\circ}}$. We shall define a subclass $\mathscr{V}$ of the regular closed subsets of $\mathbb{R}^{d}$. Let $\mathscr{D}$ be the collection of non-empty, open, convex, relatively compact sets. Then the class $\mathscr{C}$ of closures of sets in $\mathscr{D}$

$$
\mathscr{C}=\{\bar{D}: D \in \mathscr{D}\}
$$

is the class of convex bodies (convex compact sets with non-empty interior). Let

$$
\mathscr{E}=\mathscr{D} \cup \mathscr{C}
$$

and let $\mathscr{W}$ be the algebra generated by $\mathscr{E}$, i.e. by finite intersections, unions and differences of subsets of $\mathscr{E}$.

\section{Definition 1.5}

$$
\mathscr{V}=\left\{V \in \mathscr{W}: \overline{V^{\circ}}=V\right\} .
$$

An element of $\mathscr{V}$ will be called a regular set.

Any $S \in \mathscr{V}$ is compact and satisfies $\mathscr{C}^{d-1}(\partial S)<\infty$; see Appendix A.

Definition 1.6. For $S \in \mathscr{V}$ and $l \in \mathscr{L}$, write $n(l \cap S)$ for the number of components of $l \cap S$, and $\sigma(l \cap S)$ for the length (one-dimensional Hausdorff measure) of $l \cap S$. 
The following is an adaptation of a standard result in integral geometry (e.g. Santaló (1976), pp. 29, 31, 234 and Federer (1969), pp. 173, 258, 294). The proof is in Appendix A.

Lemma 1.7. With respect to the usual $\sigma$-algebra on $\mathscr{L}$, we have for $S \in \mathscr{V}$

(a) $\sigma(l \cap S)$ is a measurable function of $l \in \mathscr{L}$, and

$$
\int_{\mathscr{L}} \sigma(l \cap S) d \mu(l)=d \kappa_{d} \lambda(S)
$$

(b) $n(l \cap S)$ is a measurable function of $l \in \mathscr{L}$ and

$$
\int_{\mathscr{L}} n(l \cap S) d \mu(l)=\frac{\kappa_{d-1}}{2} \mathscr{H}^{d-1}(\partial S)
$$

In particular, for $\mu$-almost all lines $l$, the transect $l \cap S$ is a finite union of bounded line segments.

\section{The linear scan transform}

We now define the linear scan transform of a set $S$ in terms of the onedimensional covariance function of $l \cap S$.

Definition 2.1. The linear scan transform of a regular set $S \in \mathscr{V}$ is the measurable function of $l \in \mathscr{L}$ and $t>0$ defined almost everywhere by

$$
G_{l \cap S}(t)=-\frac{d}{d t} C_{l \cap S}(t)
$$

That $G_{l \cap S}$ is defined for almost all $(l, t)$ will be shown below. The following properties are immediate.

\section{Lemma 2.2}

(a) If $l \cap S=\varnothing$, then $C_{l \cap S} \equiv G_{l \cap S} \equiv 0$.

(b) For a compact convex set $K$ the intersection $l \cap K$ is either empty or a compact interval of length $\sigma(l \cap K) \geqq 0$ in which case $C_{l \cap K}(t)=(\sigma(l \cap K)-t)_{+}$and

$$
G_{l \cap K}(t)=\mathbb{1}\{\sigma(l \cap K)>t\}= \begin{cases}1 & \text { if } \sigma(l \cap K)>t \\ 0 & \text { otherwise }\end{cases}
$$

(c) $G_{l \cap s}(t)=G_{l \cap s}(t)=0$ for all $t \geqq \operatorname{diam}(S)$.

According to Lemma 1.7, $l \cap S$ is a finite union of line segments for almost all lines $l$, so that we may compute $C_{l \cap S}, G_{l \cap S}$. 
Lemma 2.3. Represent $l \cap S$ isometrically as a subset of $\mathbb{R}$ :

$$
\ln S=\bigcup_{i=1}^{n}\left[x_{2 i-1}, x_{2 i}\right]
$$

where $x_{1}<x_{2}<\cdots<x_{2 n} \in \mathbb{R}$ are the coordinates of the endpoints of the line segments (with respect to an arbitrary origin on l). Then

$$
\begin{aligned}
& C_{l \cap S}(t)=\sum_{k=1}^{2 n} \sum_{i=1}^{2 n}(-1)^{i+k+1}\left(x_{k}-x_{i}-t\right)_{+} \\
& G_{l \cap S}(t)=\sum_{k=1}^{2 n} \sum_{i=1}^{2 n}(-1)^{i+k+1} \mathbf{1}\left\{x_{k}-x_{i}>t\right\}
\end{aligned}
$$

for all $t>0$ except those satisfying $t=x_{k}-x_{i}$ for some $i \neq k$. This representation is independent of the choice of the origin and orientation on $l$.

The proof is straightforward using the representation (2.1) and integration by parts.

Since (2.1) holds for $\mu$-almost all $l$, and (2.3) is valid except at a finite number of $t$ values for each fixed $l$, the linear scan transform is defined almost everywhere (with respect to $\mu \otimes \lambda_{1}$ on $\mathscr{L} \times \mathbb{R}_{+}$).

It is an interesting exercise to check that the alternating sum expression for $C_{l \cap S}(0)$ collapses to $\sigma(l \cap S)$.

\section{Identities concerning the linear scan transform}

(a) Basic relation. Here we establish a link between the set covariance function and the linear scan transform.

Proposition 3.1. For $S \in \mathscr{V}$ and $y \in \mathbb{R}^{d}$

$$
C_{S}(y)=\int_{l_{\omega}} \int_{\|y\|}^{\infty} G_{\left(l_{\omega}+u\right) \cap s}(s) d s d \mathscr{P}^{d-1}(u),
$$

where $\omega=(1 /\|y\|) y, l_{\omega}$ is the line through 0 with direction $\omega$, and $l_{\omega}^{+}$is the orthogonal complement of $l_{\omega}$.

Proof. Let $\omega=y /\|y\| \in S^{d-1}$ and set $t=\|y\|$. By definition

$$
C_{S}(y)=C_{S}(t \omega)=\int_{\mathbb{R}^{d}} 1_{S}(x) 1_{S}(x+t \omega) d x .
$$

Decomposing $x=u+v \omega$ where $v \in \mathbb{R}$ and $u \in l_{\omega}^{\perp}$ the right-hand side of (3.1) becomes

$$
\int_{l_{\omega}} \int_{\mathbb{R}} 1_{S}(u+v \omega) 1_{S}(u+(v+t) \omega) d v d \mathscr{\ell ^ { d - 1 }}(u)=\int_{l^{\frac{1}{\omega}}} C_{\left(l_{\omega}+u\right) \cap S}(t) d \mathscr{C} \ell^{d-1}(u) .
$$


The proposition follows since $C_{l \cap S}(t)=\int_{t}^{\infty} G_{l \cap S}(s) d s$.

(b) Stereological relations. In this subsection we first establish a connection between the linear scan transform and the variables $n(l \cap S)$ and $\left[\sigma(l \cap S)^{k}\right]$.

Definition 3.2. (Miles (1983)). Let $S \in \mathscr{V}$ and $l \in \mathscr{L}$. The $k$-linc (for ' $k$ th order line section of non-convex domain') of the transect $l \cap S$ is

$$
\left[\sigma(l \cap S)^{k}\right]=\sum_{i=1}^{2 n(l \cap S)-1} \sum_{j=i+1}^{2 n(l \cap S)}(-1)^{i+j+1}\left(x_{j}-x_{i}\right)^{k}
$$

where $x_{1}, x_{2}, \cdots$ are the ordered endpoints of intercepted intervals as before and $k \geqq 0$ is an integer.

By Lemma 1.7, $\left[\sigma(l \cap S)^{k}\right]$ is well defined for almost all lines. The $k$-linc is of stereological importance, especially when $d=2$ and $k=3$ and also when $d=3$ and $k=4$ (see Miles (1983) and Jensen and Gundersen (1985)).

Lemma 3.3. For $S \in \mathscr{V}$ we have for $\mu$-almost all $l$
(a) $n(l \cap S)=G_{l \cap S}(0)=\left[\sigma(l \cap S)^{0}\right]$.
(b) $\left[\sigma(l \cap S)^{k}\right]=k \int_{0}^{\infty} t^{k-1} G_{l \cap S}(t) d t \quad$ for $k \geqq 1$.
(c) $\left[\sigma(l \cap S)^{k}\right]=k(k-1) \int_{0}^{x} t^{k-2} C_{l \cap S}(t) d t \quad$ for $k \geqq 2$.

For the proof observe that by Lemma $1.7, n(l \cap S)<\propto$ for almost all lines. For those lines, the $k$-linc of $S$ is well defined and the linear scan transform is defined for almost all lines. Consider those lines in $\mathscr{L}$ such that $n(l \cap S)<\infty$ and such that $G_{l \cap S}$ is well defined. Use relation (2.3) to prove (a) and (b); then (c) follows from (b) by integration by parts.

Examples 3.4

(A) If $K$ is convex,

$$
\begin{aligned}
{\left[\sigma(l \cap K)^{k}\right] } & =\int_{0}^{\infty} k t^{k-1} 1_{[0, \sigma(l \cap K)]}(t) d t \\
& =\int_{0}^{\sigma(l \cap K)} k t^{k-1} d t \\
& =\sigma(l \cap K)^{k} .
\end{aligned}
$$

(B) For $k=1$,

$$
\left[\sigma(l \cap S)^{1}\right]=\int_{0}^{\infty} G_{l \cap s}(t) d t=C_{l \cap S}(0)=\sigma(l \cap S),
$$

by part (a) of Lemma 1.2 . 
(C) From (B) we obtain

$$
\sigma(l \cap S)=\int_{0}^{\infty} G_{l \cap S}(t) d t
$$

for almost all $l$. Consequently

$$
\int_{\mathscr{L}} \int_{0}^{\infty} G_{l \cap S}(t) d t d \mu(l)=\int_{\mathscr{L}} \sigma(l \cap S) d \mu(l)=d \kappa_{d} \lambda(S),
$$

using Lemma 1.7.

Proposition 3.5. For $f: \mathbb{R}_{+} \rightarrow \mathbb{R}$ integrable on compact sets and $S \in \mathscr{V}$

$$
\begin{aligned}
\int_{S} \int_{S} f(\|u-v\|) d u d v & =\int_{\mathscr{L}} \int_{\mathbb{R}}|t|^{d-1} f(|t|) C_{l \cap S}(t) d t d \mu(l) \\
& =2 \int_{\mathscr{L}} \int_{0}^{\infty} t^{d-1} f(t) C_{l \cap S}(t) d t d \mu(l) \\
& =2 \int_{\mathscr{L}} \int_{0}^{\infty} F_{d-1}(t) G_{l \cap S}(t) d t d \mu(l)
\end{aligned}
$$

where $F_{d-1}(t)=\int_{0}^{t} s^{d-1} f(s) d s$.

The proof is an application of Lemma 1.4 and Borel's overlap formula (1.1).

From Proposition 3.5 and part (b) of Lemma 3.3, we obtain a generalisation of Crofton's formula, relating chord length and interpoint distance for non-convex sets, cf. Santaló (1976), p. 238, (14.25) and Miles (1979), (1985).

Corollary 3.6. For $S \in \mathcal{V}$ and $k \geqq d-1$

$$
\int_{\mathscr{L}}\left[\sigma(l \cap S)^{k}\right] d \mu(l)=\frac{k(k-1)}{2} \int_{S} \int_{S}\|u-v\|^{k-d-1} d u d v .
$$

Remark. Taking $k=d+1$ in Corollary 3.6 or $f \equiv 1$ in Proposition 3.5 we get

$$
\begin{aligned}
\lambda(S)^{2} & =\frac{2}{d(d+1)} \int_{\mathscr{L}}\left[\sigma(l \cap S)^{d+1}\right] d \mu(l) \\
& =\frac{2}{d} \int_{\mathscr{L}} \int_{0}^{\infty} t^{d} G_{l \cap S}(t) d t d \mu(l) .
\end{aligned}
$$

Finally, there is a connection with the chord length distribution

$$
F_{S}(x)=\boldsymbol{P}\{l \in \mathscr{L}: \sigma(l \cap S) \leqq x\}
$$

for a convex set $S$. Here $\boldsymbol{P}$ is the uniform distribution on the set $[S]$ of lines that intersect $S$, defined by $\boldsymbol{P}(U)=\mu(U \cap[S]) / \mu([S])$, i.e. $\boldsymbol{P}$ is the invariant measure $\mu$ restricted to $[S]$ and normalised to have unit total mass. 
Recall from Lemma 2.2(b) that $1\{\sigma(l \cap S)>x\}=G_{l \cap S}(x)$ when $S$ is convex. Hence

$$
\boldsymbol{P}\{\sigma(l \cap S) \leqq x\}=1-\frac{1}{\mu(S)} \int_{\mathscr{L}} G_{l \cap S}(x) d \mu(l) .
$$

Comparing this with results of Waksman and Pohl, we conclude that $\int_{\mathscr{L}} G_{l \cap S}(x) d \mu(l) \propto a_{S}(x)$, the associated function to $S$ defined by Pohl (1980). This was used by Waksman (1985) to partially solve the problem of characterising convex plane polygons by their chord length distributions. See also Cabo (1989).

(c) Glance functions. Waksman (1987) considered a class of open subsets $T \subset \mathbb{R}^{d}$, with diameters bounded by a fixed constant $D$, having $C^{2}$ boundaries made up of finitely many arcs on which the curvature does not change sign. He introduced the glance function which in our notation is

$$
H_{l \cap T}(t):=\sum_{i=1}^{2 n-1} \sum_{j=i+1}^{2 n}(-1)^{i+j+1} 1\left\{x_{j}-x_{i} \leqq t\right\} .
$$

Clearly

$$
H_{l \cap S}(t)=n(l \cap S)-G_{l \cap S}(t)
$$

so that our relations $n(l \cap S)=G_{l \cap S}(0)$ and $\sigma(l \cap S)=\int_{0}^{\infty} G_{l \cap S}(t) d t$ are equivalent to Waksman's results $n(l \cap S)=H_{l \cap S}(D)$ and

$$
\sigma(l \cap S)=n(l \cap S) . D-\int_{0}^{D} H_{l \cap S}(t) d t .
$$

Our change of definition makes the following section possible.

\section{Metrics}

(a) A new metric for sets

Definition 4.1. For $S, T \in \mathscr{V}$, let

$$
\begin{aligned}
\eta(S, T) & =\int_{\mathscr{L}}\left\|G_{l \cap S}-G_{l \cap T}\right\|_{1} d \mu(l) \\
& =\int_{\mathscr{L}} \int_{0}^{\infty}\left|G_{l \cap S}(t)-G_{l \cap T}(t)\right| d t d \mu(l) .
\end{aligned}
$$

This is a modification of a metric defined by Waksman (1987) in terms of $H_{I \cap S}$; the two metrics are not equivalent.

Measurability and integrability of $G_{l \cap S}(t)$ are proved in Appendix B; this ensures that $\eta$ is well defined.

Proposition 4.2. $\eta$ is a metric on $\mathscr{V}$. 
Proof. Since $\eta$ is defined as the $L^{1}$ distance between linear scan transforms, the only property to check is that $\eta(S, T)=0$ implies $S=T$. Observe that

$$
\begin{aligned}
\int_{\mathscr{L}}|\sigma(l \cap S)-\sigma(l \cap T)| d \mu(l) & =\int_{\mathscr{L}}\left|\int_{0}^{\infty} G_{l \cap S}(t) d t-\int_{0}^{\infty} G_{l \cap T}(t) d t\right| d \mu(l) \\
& \leqq \int_{\mathscr{L}} \int_{0}^{\infty}\left|G_{l \cap S}(t)-G_{l \cap T}(t)\right| d t d \mu(l) \\
& =\eta(S, T) \\
& =0 .
\end{aligned}
$$

Thus $\eta(S, T)=0$ implies

$$
\sigma(l \cap S)=\sigma(l \cap T), \quad \text { for } \mu \text {-almost lines } l .
$$

Consequently the Radon transforms of the indicator functions of $S$ and $T$ are equal for almost all lines. By Helgason (1980), Proposition I.7.5, p. 52 the indicator functions are almost everywhere equal, i.e.

$$
\lambda(S \triangle T)=0 .
$$

Since $S$ and $T$ are regular closed sets, a standard argument yields $S=T$.

Examples 4.3

(A) If $K_{1}, K_{2} \in \mathscr{V}$ are both convex,

$$
\eta\left(K_{1}, K_{2}\right)=\int_{\mathscr{L}}\left|\sigma\left(l \cap K_{1}\right)-\sigma\left(l \cap K_{2}\right)\right| d \mu(l),
$$

the $L^{1}$ distance between their Radon transforms. This follows from Lemma 2.2(b) since

$$
\begin{aligned}
\left\|G_{l \cap K_{1}}-G_{l \cap K_{2}}\right\|_{1} & =\int_{0}^{\infty}\left|1_{\left[0, \sigma\left(l \cap K_{1}\right)\right]}(t)-1_{\left[0, \sigma\left(l \cap K_{2}\right)\right]}(t)\right| d t \\
& =\left|\sigma\left(l \cap K_{2}\right)-\sigma\left(l \cap K_{1}\right)\right| .
\end{aligned}
$$

(B) If $K_{1}, K_{2}$ are convex and $K_{1} \subseteq K_{2}$ then

$$
\eta\left(K_{1}, K_{2}\right)=d_{\kappa_{d}}\left\{\lambda\left(K_{2}\right)-\lambda\left(K_{1}\right)\right\} .
$$

This follows from (1.4) together with the previous example since $\sigma\left(l \cap K_{2}\right)$ $\sigma\left(l \cap K_{1}\right) \geq 0$.

There appears to be no simple general expression for $\eta\left(K_{1}, K_{2}\right)$. For instance, the other relatively simple case of two disjoint convex sets needs results related to the Sylvester problem (see Santaló (1976), p. 63-65). However there is a simple upper bound in general. 
Lemma 4.4. For $S, T \in \mathscr{V}$

$$
\begin{aligned}
\eta(S, T) & \leqq \int_{\mathscr{L}}\left\|G_{l \cap S}\right\|_{1} d \mu(l)+\int_{\mathscr{L}}\left\|G_{l \cap T}\right\|_{1} d \mu(l) \\
& \leqq \operatorname{diam}(S) \text { length }(\partial S)+\operatorname{diam}(T) \text { length }(\partial T) .
\end{aligned}
$$

Proof. The first inequality follows from the triangle inequality for the $L^{1}$ norm. Clearly

$$
\left|G_{l \cap S}(t)\right| \leqq G_{l \cap S}(0)=n(l \cap S) \quad \text { for all } t .
$$

Hence $\left\|G_{l \cap S}\right\|_{1} \leqq n(l \cap S) \operatorname{diam}(S)$. This yields the lemma by (1.5), see Santaló (1976), p. 31.

Remark. The first upper bound in (4.3) is sharp in the sense that it equals the supremum of $\eta\left(S, T^{\prime}\right)$ over all $T^{\prime}$ congruent to $T$. This may be seen by considering translations of $T$ by vectors $x_{n} \in \mathbb{R}^{d}$ with $\left\|x_{n}\right\| \rightarrow \infty$.

(b) Connection with the Hausdorff distance

Definition 4.5. The Hausdorff distance between two non-empty sets $A, B \subset \mathbb{R}^{d}$ is

$$
\begin{aligned}
\mathscr{H}(A, B) & =\max \left\{\sup _{a \in A} \inf _{b \in B}\|a-b\|, \sup _{b \in B} \inf _{a \in A}\|a-b\|\right\} \\
& =\inf \left\{r>0: A \subset B^{r} \text { and } B \subset A^{r}\right\},
\end{aligned}
$$

where the outer parallel set or dilation of $B$ by $r>0$ is

$$
B^{r}=\left\{x \in \mathbb{R}^{d}: \inf _{b \in B}\|x-b\| \leqq r\right\} .
$$

We also define the inner parallel set or erosion

$$
B^{-r}=\left\{x \in B: \inf _{c \in B^{c}}\|x-c\| \geqq r\right\} .
$$

The following example shows that the two metrics do not generate the same topology on $\mathscr{V}$.

\section{Example 4.6. Define}

2) -

$$
X_{n}=B(0,1) \cup B\left(x, \frac{1}{n}\right) \quad \text { where } n \geqq 1 \text { and }\|x\|=3 \text {. }
$$

In the Hausdorff metric $X_{n}$ converges to $B(0,1) \cup\{x\}$. However, in $\eta$ it converges to $B(0,1)$ by the following argument. Split the integral

pper

$$
\eta\left(X_{n}, B(0,1)\right)=\int_{\mathscr{L}}\left\|G_{l \cap X_{n}}-G_{l \cap B(0,1)}\right\|_{1} d \mu(l)
$$


into three terms corresponding to the domains $[B(0,1)] \backslash[B(x, 1 / n)],[B(x, 1 / n)] \backslash$ $[B(0,1)]$ and $[B(0,1)] \cap[B(x, 1 / n)]$ respectively. The first term is zero because $G_{l \cap X_{n}} \equiv G_{l \cap B(0,1)}$ on the domain of integration. The second term is

$$
\int_{[B(x, 1 / n) \backslash[B(0,1)]}\left\|G_{l \cap B(x, 1 / n)}\right\|_{1} d \mu(l)=\int_{[B(x, 1 / n)] \backslash B(0,1)]} \sigma\left(\ln B\left(x, \frac{1}{n}\right)\right) d \mu(l)
$$

by convexity and Example 3.4(B). But the right side is dominated by

$$
\int_{[B(x, 1 / n)]} \sigma\left(\ln B\left(x, \frac{1}{n}\right)\right) d \mu(l)=\frac{d \kappa_{d}^{2}}{n^{d}} \rightarrow 0 \quad \text { as } n \rightarrow \infty .
$$

The third term tends to zero since the integrand is bounded above by 10 using (4.4), and $\mu([B(0,1)] \cap B(x, 1 / n)) \rightarrow \mu([B(0,1)] \cup[\{x\}])=0$ as $n \rightarrow \infty$. Hence $X_{n} \rightarrow$ $B(0,1)$ in $\eta$.

Theorem 4.7. The metrics $\eta$ and $\mathscr{H}$ are topologically equivalent on the space $\mathscr{C}$ of convex bodies.

The proof is divided into several parts.

Proposition 4.8. Let $K_{n}, K \in \mathscr{C}$. If $\mathscr{H}\left(K_{n}, K\right) \rightarrow 0$ then $\eta\left(K_{n}, K\right) \rightarrow 0$.

Proof. For fixed $n$, if $\mathscr{H}\left(K, K_{n}\right)<\delta$ then by definition

$$
K \subset\left(K_{n}\right)^{\delta} \quad \text { and } \quad K_{n} \subset K^{\delta}
$$

where $K^{\delta}$ is defined in 4.5. Since $K_{n}$ is convex, $\left(\left(K_{n}\right)^{\delta}\right)^{-\delta}=K_{n}$ so

$$
K^{-\delta} \subset K_{n} \subset K^{\delta} \text {. }
$$

Hence for all $l \in \mathscr{L}$

$$
\sigma\left(K^{-\delta} \cap l\right) \leqq \sigma\left(K_{n} \cap l\right) \leqq \sigma\left(K^{\delta} \cap l\right) .
$$

But also $K^{-\delta} \subset K \subset K^{\delta}$ so that $\sigma(K \cap l)$ is also sandwiched between these limits. Hence

$$
\left|\sigma(K \cap l)-\sigma\left(K_{n} \cap l\right)\right| \leqq \sigma\left(K^{\delta} \cap l\right)-\sigma\left(K^{-\delta} \cap l\right) .
$$

Integrating as in Example 4.3(B) we obtain

$$
\eta\left(K, K_{n}\right) \leqq d \kappa_{d}\left[\lambda\left(K^{\delta}\right)-\lambda\left(K^{-\delta}\right)\right] .
$$

The right-hand side converges to zero as $\delta \rightarrow 0$ since the boundary of a convex set has Lebesgue measure zero. The result follows. 
Remark. Using the Steiner formula (Santaló (1976), p. 220) one can easily obtain the explicit bound (for convex $S, T$ )

$$
\begin{aligned}
\eta(S, T) \leqq & 2 d \kappa_{d} \sum_{s=1}^{d}\left(\begin{array}{l}
d \\
s
\end{array}\right) W_{s}(S) \mathscr{H}(S, T)^{s} \\
& +d \kappa_{d} \sum_{s=1}^{d}\left(\begin{array}{c}
d \\
s
\end{array}\right) \sum_{k=0}^{d-s}\left(\begin{array}{c}
d-s \\
k
\end{array}\right) W_{k}(S) \mathscr{H}(S, T)^{s+k}
\end{aligned}
$$

where $W_{k}(\cdot)$ is the $k$ th quermassintegral (Santaló (1976), p. 217).

For the converse of Proposition 4.8 we need the following.

Definition 4.9 (see Eggleston (1958)). Let $K \in \mathscr{C}$.

(i) The inradius $r(K)$ of $S$ is the supremum of the radii of all balls contained in $K$.

(ii) The minimal width $w(K)$ of $K$ is the infimum of the distances between all pairs of parallel supporting hyperplanes of $K$.

Remark. Clearly $r(K)<\epsilon$ iff $K^{-\epsilon}=\varnothing$, where $K^{-\epsilon}$ is the erosion defined in 4.5.

Lemma 4.10. Suppose $\eta\left(K_{n}, K\right) \rightarrow 0$ and $\bigcup_{n=1}^{\infty} K_{n}$ is unbounded. Then $r\left(K_{n}\right) \rightarrow 0$ as $n \rightarrow \infty$.

Proof. Suppose $\cup K_{n}$ is unbounded. Choosing a subsequence if necessary, we may find $x_{n} \in K_{n}$ such that $0<\left\|x_{n}\right\| \uparrow \propto$.

We prove the result by contradiction. Suppose (possibly for a subsequence) $r\left(K_{n}\right) \geqq \epsilon$ for all $n$ where $\epsilon>0$. We claim that $D\left(K, K_{n}^{-\epsilon / 2}\right) \rightarrow \infty$, where

$$
D(S, T):=\sup _{y \in T} d(y, S)=\sup _{y \in T} \inf _{x \in S}\|x-y\| .
$$

For, either $D\left(K, K_{n}^{-\epsilon}\right) \rightarrow \infty$ (which implies the claim) or (possibly for a subsequence) $\sup _{n} D\left(K, K_{n}^{-\epsilon}\right)=M<\infty$. Assume the latter case. For every $n$ we can find $y_{n} \in K_{n}^{-\epsilon}$ such that $d\left(y_{n}, K\right) \leqq M$, so that $B\left(y_{n}, \epsilon\right) \subset K^{M+\epsilon}$.

Put $C_{n}:=\operatorname{co}\left(B\left(y_{n}, \epsilon\right) \cup\left\{x_{n}\right\}\right)$. Then $C_{n} \subseteq K_{n}$. Defining $z_{n}=\left(x_{n}+y_{n}\right) / 2$ simple trigonometry shows that

$$
B_{n}=B\left(z_{n}, \epsilon / 2\right) \subset C_{n}
$$

Hence $z_{n} \in K_{n}^{-\epsilon / 2}$. Now

$$
\begin{aligned}
d\left(z_{n}, K\right) & \geqq\left\|z_{n}-y_{n}\right\|-d\left(y_{n}, K\right) \\
& \geqq\left\|z_{n}-y_{n}\right\|-M \\
& =\frac{1}{2}\left\|x_{n}-y_{n}\right\|-M \\
& \rightarrow \infty .
\end{aligned}
$$

Thus $D\left(K, K_{n}^{-\epsilon / 2}\right) \rightarrow \infty$, proving the claim. 
Consequently we can find balls $B\left(z_{n}, \epsilon / 2\right)=B_{n} \subset K_{n}$ such that $\left\|z_{n}\right\| \rightarrow \infty$. Now

$$
\begin{aligned}
\eta\left(K_{n}, K\right) & \geqq \int_{\left.\left[B_{n}\right] \backslash K\right]} \sigma\left(l \cap B_{n}\right) d \mu(l) \\
& =\int_{\left[B_{n}\right]} \sigma\left(l \cap B_{n}\right) d \mu(l)-\int_{\left[B_{n}\right] \cap[K]} \sigma\left(l \cap B_{n}\right) d \mu(l) \\
& \geqq d \kappa_{d} \lambda\left(B_{n}\right)-\epsilon \mu\left(\left[B_{n}\right] \cap[K]\right),
\end{aligned}
$$

by (1.4) and the fact that $\sigma\left(l \cap B_{n}\right) \leq \epsilon$. If $B=B(x$, diam $(K))$ is the circumsphere of $K$, then we have

$$
\mu\left(\left[B_{n}\right] \cap[B]\right)=c\left(\frac{1}{2} \operatorname{diam}(K), \epsilon / 2,\left\|z_{n}-x\right\|\right),
$$

where $c\left(r_{1}, r_{2}, s\right)$ is the measure of all lines intersecting two disjoint balls of radii $r_{1}$, $r_{2}$ with midpoints separated by a distance $s$. By standard integral geometric arguments it can be shown that for fixed $r_{1}, r_{2}, c\left(\dot{r}_{1}, r_{2}, s\right) \rightarrow 0$ as $s \rightarrow \infty$. Hence $\mu\left(\left[B_{n}\right] \cap[B]\right) \rightarrow 0$, i.e.

$$
\lim _{n \rightarrow \infty} \eta\left(K_{n}, K\right)>\kappa_{d}(\epsilon / 2)^{d}>0 .
$$

This contradiction proves the lemma.

Lemma 4.11. (Eggleston, 1958, p. 112). For a compact convex set $K$

$$
r(K) \geqq c_{d} \cdot w(K)
$$

where $c_{d}$ is a constant depending only on the dimension $d$.

Lemma 4.12. Let $K \in \mathscr{C}$. Then

$$
\sup _{\delta>0} \inf _{w(L)<\delta} \eta(K, L)>0
$$

where the infimum ranges over $L \in \mathscr{C}$. In particular, any sequence $K_{n}$ satisfying $w\left(K_{n}\right) \rightarrow 0$ has $\lim \sup _{n} \eta\left(K, K_{n}\right)>0$ for all $K \in \mathscr{C}$.

Proof. Fix $0<\alpha<r(K)$. Observe that $\lambda\left(K^{-\alpha}\right) \neq \varnothing$. Fix $0<\eta<\frac{2}{3} \kappa_{d} \lambda\left(K^{-\alpha}\right)$. Then

$$
\lambda\left(K^{-\alpha}\right)>\frac{\eta}{\frac{2}{3} \kappa_{d}} .
$$

Now choose

and let $L \in \mathscr{C}$ with $\omega(L)<\delta$.

$$
\delta<\min \left(\alpha, \frac{\frac{2}{3} \kappa_{d} \lambda\left(K^{-\alpha}\right)-\eta}{2 \mu([K])}\right)
$$

Choose a direction $\theta_{\min }^{\perp}$ such that the two parallel support hyperplanes of $L$ 
normal to this direction are at the minimum distance $w(L)$ apart. Write $W$ for the region bounded by these two supporting hyperplanes, and let $\mathscr{L}_{\alpha}$ be the set of lines $l$ intersecting $K^{-\alpha}$ whose directions $\theta(l)$ make an angle $\varangle\left(\theta(l), \theta_{\min }^{\perp}\right)$ with $\theta_{\min }^{\perp}$ that lies in the interval $\left(-\frac{1}{3} \pi, \frac{1}{3} \pi\right)$ :

$$
\mathscr{L}_{\alpha}:=\left\{l \in\left[K^{-\alpha}\right]: \varangle\left(\theta(l), \theta_{\min }^{\perp}\right) \in\left(-\frac{1}{3} \pi, \frac{1}{3} \pi\right)\right\} .
$$

Let $l \in \mathscr{L}_{\alpha}$. Then by assumption

$$
\sigma(l \cap W)=\frac{w(L)}{\cos \left(\varangle\left(\theta, \theta_{\min }^{\perp}\right)\right)}<2 w(L),
$$

where we use the fact that $\cos \phi>\frac{1}{2}$ if $\phi \in\left(-\frac{1}{3} \pi, \frac{1}{3} \pi\right)$. Thus also

$$
\sigma(l \cap L)<2 w(L) .
$$

Furthermore, observe that for lines intersecting $K^{-\alpha}$,

$$
\sigma(l \cap K) \geqq 2 \alpha .
$$

Hence

$$
\sigma(l \cap K) \geqq 2 \alpha>2 \delta>2 w(L)>\sigma(l \cap L) \quad \text { for } l \in \mathscr{L}_{\alpha} .
$$

Consequently

$$
\begin{aligned}
\eta(K, L) & \geqq \int_{\mathscr{L}_{\alpha}}|\sigma(l \cap K)-\sigma(l \cap L)| d \mu(l) \\
& =\int_{\mathscr{L}_{\alpha}} \sigma(l \cap K) d \mu(l)-\int_{\mathscr{L}_{\alpha}} \sigma(l \cap L) d \mu(l) .
\end{aligned}
$$

By part (a) of Lemma 1.7 and the definition of $\mathscr{L}_{\alpha}$

$$
\int_{\mathscr{L}_{\alpha}} \sigma(l \cap K) d \mu(l) \geqq \int_{\mathscr{L}_{\alpha}} \sigma\left(l \cap K^{-\alpha}\right) d \mu(l)=\frac{2}{3} \kappa_{d} \lambda\left(K^{-\alpha}\right) .
$$

For the second integral we have by (4.7)

$$
\begin{aligned}
\int_{\mathscr{L}_{\alpha}} \sigma(l \cap L) d \mu(l) & <2 w(L) \mu\left(\mathscr{L}_{\alpha}\right) \\
& \leqq 2 w(L) \mu([K]) .
\end{aligned}
$$

Summarising

$$
\begin{aligned}
\eta(K, L) & \geqq \frac{2}{3} \kappa_{d} \lambda\left(K^{-\alpha}\right)-2 w(L) \mu([K]) \\
& >\frac{2}{3} \kappa_{d} \lambda\left(K^{-\alpha}\right)-2 \delta \mu([K]) \quad \text { by assumption } \\
& >\eta, \quad \text { by }(4.7) .
\end{aligned}
$$

Thus $\inf _{w(L)<\delta} \eta(K, L) \geq \eta$; but this implies the lemma. 
Proposition 4.13. Let $K, K_{n}$ be in $\mathscr{C}$ with $\eta\left(K_{n}, K\right) \rightarrow 0$. Then $\bigcup_{n} K_{n}$ is bounded.

Proof. Assume $\eta\left(K_{n}, K\right) \rightarrow 0$ and suppose to the contrary that $\bigcup_{n=1}^{\infty} K_{n}$ is unbounded. By Lemma 4.10, $r\left(K_{n}\right) \rightarrow 0$. Then by Lemma 4.11, $w\left(K_{n}\right) \rightarrow 0$. But by Lemma $4.12, \lim \sup _{n} \eta\left(K_{n}, K\right)>0$. This contradiction implies that $\cup_{n=1}^{\infty} K_{n}$ is bounded, proving the proposition.

Finally we are able to state the converse to Proposition 4.8.

Proposition 4.14. Let $K_{n}, K \in \mathscr{C}$. If $\eta\left(K_{n}, K\right) \rightarrow 0$ then $\mathscr{H}\left(K_{n}, K\right) \rightarrow 0$.

Proof. Assume to the contrary that $K_{n}$ tends to $K$ in $\eta$, but $n o t$ in $\mathscr{H}$. Then for all $\epsilon>0$, there is a subsequence, $K_{n_{i}}$ say, such that

$$
\mathscr{H}\left(K_{n_{i}}, K\right)>\epsilon \quad \text { for all } i \text {. }
$$

Since by assumption $\eta\left(K_{n}, K\right) \rightarrow 0$, Proposition 4.13 yields that $\cup_{i} K_{n_{i}}$ is bounded. However by Blaschke's selection theorem (see Eggleston (1958)) there is a sub-subsequence, $K_{n_{i j}}$ say, that does converge in $\mathscr{H}$. Suppose its limit is $K^{*}$ :

$$
\mathscr{H}\left(K_{n_{i}}, K^{*}\right) \rightarrow 0, \quad j \rightarrow \infty .
$$

But then

$$
\eta\left(K, K^{*}\right) \leqq \eta\left(K, K_{n_{i}}\right)+\eta\left(K_{n_{i,}}, K^{*}\right) \rightarrow 0,
$$

by the assumption and Proposition 4.8. That is, $\eta\left(K, K^{*}\right)=0$ and hence $K=K^{*}$, i.e.

$$
K_{n_{i}} \stackrel{\mathscr{H}}{\rightarrow} K \text {. }
$$

This contradicts (4.9), establishing the proposition.

Finally, Theorem 4.7 follows immediately from Propositions 4.8 and 4.11 .

\section{Continuity results}

(a) Volume

Lemma 5.1. The mapping $S \mapsto \lambda(S)$ is Lipschitz-continuous with constant 1 on $(\mathscr{V}, \eta)$

Proof. By Proposition 3.1

$$
\begin{aligned}
|\lambda(S)-\lambda(T)| & =\left|C_{S}(0)-C_{T}(0)\right| \\
& \leqq \int_{\mathscr{L}} \int_{0}^{\infty}\left|G_{l \cap S}(t)-G_{l \cap T}(t)\right| d t d \mu(l) \\
& =\eta(S, T) .
\end{aligned}
$$

(b) The set covariance function. As a corollary to the equivalence of $\eta$ and $\mathscr{H}$ on the collection of convex bodies, we obtain the following. 
Corollary 5.2. Let $K, K_{n} \in \mathscr{C}$ and suppose $\mathscr{H}\left(K_{n}, K\right) \rightarrow 0$. Then

$$
C_{K_{n}}(y) \rightarrow C_{K}(y) \text { pointwise. }
$$

Proof. Fix $y \in \mathbb{R}^{d}$ and write $C_{K}(y)=\int_{\mathbb{R}^{d}} 1_{K_{n}}(x) 1_{T_{y} K_{n}}(x) d x$. Since $\mathscr{H}\left(K_{n}, K\right) \rightarrow 0$ we have

$$
K^{-\epsilon_{n}} \subset K_{n} \subset K^{\epsilon_{n}}
$$

where $\epsilon_{n} \rightarrow 0$ as $n \rightarrow \infty$. Hence

$$
1_{K^{-\epsilon_{n}}}(x) \leqq 1_{K_{n}}(x) \leqq 1_{K^{\epsilon_{n}}}(x), \quad \forall x .
$$

Since the two bounds converge to $1_{K}(x)$ as $n \rightarrow \infty$ pointwise on $\mathbb{R}^{d} \backslash \partial K$, the same is true for $1_{K_{n}}(x)$. Dominated convergence yields

$$
C_{K_{n}}(y)=\int_{\mathbb{R}^{d}} 1_{K_{n}}(x) 1_{T_{y} K_{n}}(x) d x \rightarrow \int_{\mathbb{R}^{d}} 1_{K}(x) 1_{T_{y} K}(x) d x=C_{K}(y) .
$$

The representation of the covariance function in terms of $G$ also enables us to prove continuity of the set covariance as a function of its set argument with respect to the metric $\eta$. The following result is useful.

Lemma 5.3. For $r>0$ and $S, T \in \mathscr{V}$

$$
\int_{\|y\|=r}\left|C_{S}(y)-C_{T}(y)\right| d \omega_{r} \leqq \eta(S, T),
$$

where $\omega_{r}$ is the spherical measure on a ball with radius $r$.

Proof. By Proposition 3.1 (using the notation introduced there)

$$
\begin{aligned}
\left|C_{S}(y)-C_{T}(y)\right| & =\left|\int_{l_{\omega}^{1}} \int_{\|y\|}^{\infty} G_{\left(l_{\omega}+\xi\right) \cap s}(t)-G_{\left(l_{\omega}+\xi\right) \cap T}(t) d t d \mathscr{H}^{d-1}(\xi)\right| \\
& \leqq \int_{l_{\omega}^{ \pm}} \int_{\|y\|}^{\infty}\left|G_{\left(l_{\omega}+\xi\right) \cap s}(t)-G_{\left(l_{\omega}+\xi\right) \cap T}(t)\right| d t d \mathscr{H}^{d-1}(\xi) \\
& \leqq \int_{l_{\omega}^{d}} \| G_{\left(l_{\omega}+\xi\right) \cap s}-G_{\left(l_{\omega}+\xi\right) \cap T \|_{1}} d \mathscr{C}^{d-1}(\xi) .
\end{aligned}
$$

Integrating over all directions yields the lemma.

Theorem 5.4. Given $M>0$ define

$$
\mathscr{V}(M):=\{S \in \mathscr{V}: \operatorname{diam}(S) \leqq M\}
$$

The mapping $S \mapsto C_{S}$ from $(\mathscr{V}(M), \eta)$ into $L^{1}\left(\mathbb{R}^{d}\right)$ is Lipschitz continuous with constant $\frac{1}{2} M^{2}$ :

$$
\left\|C_{S}-C_{T}\right\|_{1} \leqq \frac{1}{2} M^{2} \eta(S, T) \quad \text { for all } S, T \in \mathscr{V}(M)
$$


Proof. Let $S, T \in \mathscr{V}(M)$. Transforming to polar coordinates

$$
\begin{aligned}
\left\|C_{S}-C_{T}\right\|_{1} & =\int_{\mathbb{R}^{d}}\left|C_{S}(y)-C_{T}(y)\right| d \lambda(y) \\
& =\int_{0}^{\infty} r \int_{\|y\|=r}\left|C_{S}(y)-C_{T}(y)\right| d \omega_{r} d r \\
& =\int_{0}^{M} r \int_{\|y\|=r}\left|C_{S}(y)-C_{T}(y)\right| d \omega_{r} d r \\
& \leqq \eta(S, T) \int_{0}^{M} r d r \quad \text { by Lemma } 5.3 \\
& =\frac{1}{2} M^{2} \eta(S, T) .
\end{aligned}
$$

Theorem 5.5 (Matheron (1986)). Let $K \in \mathscr{C}$ be fixed. The mapping $y \mapsto C_{K}(y)$ from $\mathbb{R}^{d}$ to $\mathbb{R}_{+}$is Lipschitz continuous:

$$
\left|C_{K}(y)-C_{K}(x)\right| \leqq 2 b\|x-y\|,
$$

where $b$ is the supremum over $S^{d-1}$ of the breadth function of $K$.

Corollary 5.6. Let $K_{n}, K \in \mathscr{C}$ with $\eta\left(K_{n}, K\right) \rightarrow 0$. Then

$$
C_{K_{n}} \rightarrow C_{K} \quad \text { uniformly. }
$$

Proof. By Corollary 5.2

$$
C_{K_{n}}(y) \rightarrow C_{K}(y)
$$

Equation (5.1) holds, hence

$$
C_{K^{-\varepsilon_{n}}} \leqq C_{K_{n}} \leqq C_{K^{\varepsilon_{n}}}
$$

Observe that the sequences $\left\{C_{K^{-\varepsilon_{n}}}\right\}_{n=1}^{\infty}$ and $\left\{C_{K^{\varepsilon_{n}}}\right\}_{n=1}^{\infty}$ are both monotone. Since $\mathscr{H}\left(K^{\epsilon_{n}}, K\right)=\epsilon_{n} \downarrow 0$, Theorem 4.7 and Corollary 5.2 yield

$$
C_{K^{\varepsilon_{n}}}(y) \downarrow C_{K}(y), \quad \text { for } y \in \mathbb{R}^{d} .
$$

Moreover since $K^{-\epsilon_{n}} \uparrow K$ then $\mathscr{H}\left(K^{-\epsilon_{n}}, \bar{K}\right) \rightarrow 0$ because $\bar{K}$ is compact (Matheron (1975), Cor. 3, p. 13). This yields $\mathscr{H}\left(K^{-\epsilon_{n}}, K\right) \rightarrow 0$ by compactness of $K$. Thus also

$$
C_{K^{-\epsilon_{m}}} \uparrow C_{K}(y) \text {. }
$$

By Theorem 5.5, all these functions are continuous. Since they all have compact supports, Dini's theorem asserts that $C_{K^{\epsilon_{n}}}$ and $C_{K^{-\epsilon_{n}}}$ converge uniformly to $C_{K^{\text {. }}}$. The triangle inequality and other standard arguments together with (5.2) yield the claim. 


\section{Appendix A: Proof of Lemma 1.7}

To prove Lemma 1.7, recall the following concepts.

Definition A.1. Let $E \subset \mathbb{R}^{d}$. Then $E$ is $\left(\mathscr{H}^{m}, m\right)$-rectifiable if $\mathscr{H}^{m}(E)<\infty$ and there exists a set $F$ containing $\mathscr{H}^{m}$-almost all of $E$ such that $F=\bigcup_{n=1}^{\infty} F_{n}$, where each $F_{n}$ is the image of a bounded subset of $\mathbb{R}^{m}$ under a Lipschitz map. (See Federer (1969) pp. 251, 252 or Simon (1983).)

For any $\left(\mathscr{H}^{m}, m\right)$-rectifiable set $E$

$$
\mathscr{H}^{m}(E)=c(m, d) \int_{\mathscr{L}} \mathscr{H}^{m+1-d}(l \cap E) d \mu(l)
$$

for a certain constant $c(m, d)$.

It is well known that the boundary of a bounded convex set with non-empty interior in $\mathbb{R}^{d}$ is $\left(\mathscr{H}^{d-1}, d-1\right)$-rectifiable. The following result is easy to prove.

Lemma A.2. Every $W \in \mathscr{W}$ (see Section 1.2(c)) can be represented (not uniquely) as a disjoint union

$$
W=\bigcup_{j=1}^{m}\left(\left(D_{j} \cap C_{j}\right) \backslash\left(\bigcup_{k=1}^{m_{j}} E_{j k}\right)\right),
$$

where $D_{j} \in \mathscr{D}, C_{j} \in \mathscr{C}, E_{j k} \in \mathscr{E}$.

Corollary A.3. For the boundary of a set $W \in \mathscr{W}$

$$
\partial W \subset \bigcup_{j=1}^{m}\left(\partial D_{j}\right) \cup\left(\partial C_{j}\right) \cup \bigcup_{k=1}^{m_{j}}\left(\partial E_{j k}\right) .
$$

Consequently there exist $K_{i} \in \mathscr{C}$

$$
\partial W \subset \bigcup_{i=1}^{n} \partial K_{i}
$$

In particular, $\partial W$ is $\left(\mathscr{H}^{d-1}, d-1\right)$-rectifiable.

Proof of Lemma 1.7. Since $S \in \mathscr{V}, \partial S$ is $\left(\mathscr{H}^{d-1}, d-1\right)$-rectifiable, so we can apply (A.1) with $m=d-1$ and $m+1-d=0$ yielding

$$
\int_{\mathscr{L}} \mathscr{H}^{0}(l \cap \partial S) d \mu(l)=c(d-1, d) \mathscr{H}^{d-1}(\partial S) .
$$

In particular $\mu(\mathscr{L}(5)=0$ where $\mathscr{F}=\{l \in \mathscr{L}: l \cap \partial S$ is finite $\}$.

Let $\mathfrak{A}:=\left\{l \in \mathscr{L}: n(l \cap S) \neq \frac{1}{2} \mathscr{H}^{0}(\partial S \cap l)\right\}$. We need to prove $\mu(\mathfrak{A})=0$.

For any $l \in\left(5\right.$ we have $2 n(l \cap S) \leqq \mathscr{H}^{0}(\partial S \cap l)$ since every endpoint of an interval of $l \cap S$ is a point of $\partial S$. Conversely each point $x \in l \cap \partial S$ is either (a) an endpoint of an interval of $l \cap S$, (b) an isolated point of $l \cap S$, or (c) a point interior to an interval of $l \cap S$. If all $x \in l \cap \partial S$ are of type (a), clearly $n(l \cap S)=\frac{1}{2} \mathscr{H}^{0}(\partial S \cap l)$. 
Hence it suffices to prove that $\mu(\mathscr{L} \backslash \mathfrak{F})=0$ where $\mathfrak{F}=\{l \in \mathfrak{F}$ : all points of $l \cap \partial S$ are endpoints of intervals of $l \cap S\}$.

Fix $l \in \mathcal{F}$ and $x \in l \cap \partial S$. Let $\left\{K_{i}\right\}_{i}=\left\{D_{j}\right\}_{j} \cup\left\{C_{j}\right\}_{j} \cup\left\{E_{j k}\right\}_{j, k}$ be the convex sets featuring in the representation of $S$ at Lemma A.2. Observing $\partial S \subseteq \bigcup_{i} \partial K_{i}$, consider only those $\partial K_{i}$ which contain $x$. Choose a convex open neighbourhood $U$ of $x$ in $\mathbb{R}^{d}$ such that $U \cap l \cap \partial K_{i}=\{x\}$. The convex hypersurfaces $\partial K_{i}$ intersect at $x$ and divide $U \backslash \partial S$ into disjoint connected open sets $O_{j}$. For each $O_{j}$ either $O_{j} \subset S^{\circ}$ or $O_{j} \subset S^{c}$. Since $S$ is regular closed, $O_{j} \subset S^{\circ}$ for at least one $j$ and since $x \in \partial S, O_{j^{\prime}} \subset S^{c}$ for at least one $j^{\prime}$. Hence for any line $l \in \mathbb{E}$ which does not contain $x, U \cap l \cap \partial S$ consists of endpoints of intervals of $U \cap l \cap S$. But $\mu\{l \in \mathscr{L}: x \in l\}=0$, so a covering argument gives $\mu(\mathscr{L} \mathfrak{Z} \mathfrak{F})=0$, establishing the lemma.

\section{Appendix B}

Lemma B.1. For every $S \in \mathcal{V}$ the function $G_{l \cap S}(t)$ is jointly measurable in $(l, t)$ and absolutely integrable.

Proof. We first prove measurability of the transect covariance function by applying the coarea formula (Federer (1969), 3.2.22). Let $\mathbb{V}:=\mathbb{R}^{d} \times S^{d-1}$. Define $f: \vee \times \mathbb{R} \rightarrow \mathbb{R}^{d}$ by $f((x, u), t)=x+t u$ that is, $f$ maps $(x, u, t)$ onto a point at distance $t$ from $x$, lying on the line with orientation $u$ through $x$. Next define $g: \mathbb{V} \times \mathbb{R} \rightarrow$ $\mathbb{R}^{d} \times \mathbb{R}^{d}$ by $g((x, u), t)=(x, f(x, u, t))$. For any measurable set $A \subset \mathbb{R}^{d}$ with finite Lebesgue measure, the set

$$
A^{*}:=g^{-1}(A \times A)=\{(x, u, t): x \in A, f(x, u, t) \in A\}
$$

is clearly measurable. Next define $h: \mathbb{V} \rightarrow \mathscr{L}$ by $h(x, u)=\{x+a u: a \in \mathbb{R}\}$. It is simple but tedious to construct local coordinates on $\mathscr{L}$ such that $h$ is locally Lipschitz. Finally define $i: \mathbb{V} \times \mathbb{R} \rightarrow \mathscr{L} \times \mathbb{R}$ by $i((x, u), t)=(h(x, u), t)$. Then the coarea formula implies that

$$
\begin{aligned}
s(l, t): & =\int_{i^{-1}(l, t)} 1_{A^{*}}((x, u), t) d \mathscr{C}^{1}(x, u) \\
& =2 C_{l \cap A}(t)
\end{aligned}
$$

is measurable in $(l, t)$.

For sets $A \in \mathscr{V}, G_{l \cap S}(t)$ is minus the $t$ derivative of $C_{l \cap A}(t)$ and $\left|G_{l \cap S}(t)\right| \leqq$ $G_{l \cap S}(0)=n(l \cap S)$ where the bound $n(l \cap S)$ is integrable by Lemma 1.7; so $G_{l \cap S}(t)$ is measurable and absolutely integrable.

\section{Acknowledgments}

We are very grateful to Professor R. A. Vitale for suggestions leading to the proof of Theorem 4.7 and to the referee and Professors R. D. Gill and P. Groeneboom for helpful comments. This research was funded by CWI, Amsterdam. 


\section{References}

Ambartzumian, R. V. (1982) Combinatorial Integral Geometry. Wiley, New York.

BOREL, E. (1947) Principles et formules classiques du calcul des probabilités. Gauthier-Villars, Paris.

CABO, A. J. (1989) Chord length distributions and characterization problems for convex plane polygons. Master's thesis, University of Amsterdam.

CROFTON, M. W. (1885) Probability. Encyclopaedia Britannica, 9th edn, Vol. 29, pp. 768-788.

EgGleston, H. G. (1958) Convexity. Cambridge University Press.

FEDERER, H. (1969) Geometric Measure Theory. Springer-Verlag, Berlin.

GOODEY, P. AND WEIL, W. (1992) The determination of convex bodies from the mean or random sections. Math. Proc. Camb. Phil. Soc. 112, 419-430.

Helgason, S., (1980) The Radon Transform. Progress in Mathematics 5. Birkhäuser, Basel.

Jensen, E. B. AND Gundersen, H. J. G. (1985) The stereological estimation of moments particle volume. J. Appl. Prob. 22, 82-98.

LEŠANOVSKÝ, A. AND RATAJ, J. (1990) Determination of compact sets in Euclidean spaces by the volume of their dilation. Proc. Conf. DIANA III, June 1990, Math. Inst. of CŠAV, Praha 1990, pp. 165-177.

MALLOWS, C. L. and CLARK, J. M. (1970) Linear intercept distributions do not characterize plane sets. J. Appl. Prob. 7, 240-244.

Mallows, C. L. and ClaRK, J. M. (1971) Corrections to 'Linear intercept distributions do not characterize plane sets', J. Appl. Prob. 8, 208-209.

MatéRn, B. (1985) Spatial Variation. Springer-Verlag, Berlin.

MatHeron, G. (1975) Random Sets and Integral Geometry. Wiley, New York.

Matheron, G. (1986) Le covariogramme géométrique des compacts convexes de $\mathbb{R}^{2}$. Technical Report 2/86, Centre de Géostatistique, Ecole des Mines de Paris, February 1986.

Miles, R. E. (1979) Some new integral formulae, with stochastic applications. J. Appl. Prob. 16, 592-606.

MILES, R. E. (1983) Stereology for embedded aggregates of not-necessarily-convex particles. Memoirs, Vol. 6, Department of Theoretical Statistics, University of Aarhus, Aarhus, pp. 127-147.

MILES, R. E. (1985) A comprehensive set of stereological formulae for embedded aggregates of not-necessarily-convex particles. J. Microscopy 138, 115-125.

NAGEL, W. (1991) Das geometrische Kovariogramm und verwandte Größen zweiter Ordnung. Habilitationsschrift, Friedrich-Schiller-Universität Jena.

NAGEL, W. (1993) Orientation-dependent chord length distributions characterize convex polygons. $J$. Appl. Prob. 30, 730-736.

PoHL, W. F. (1980) The probability of linking of random closed curves. In Geometry Symposium

Utrecht, ed. E. Looijenga et al. pp. 113-125. Lecture Notes in Mathematics 894, Springer-Verlag, Berlin. SANTALó, L. A. (1976) Integral Geometry and Geometric Probability. Wiley, New York. SCHMITT, M. (1993). On two inverse problems in mathematical morphology. In Mathematical Morphology in Image Processing, ed. E. R. Dougherty, pp. 151-169. Marcel Dekker, New York. Simon, L. (1983) Lectures on Geometric Measure Theory. Vol. 3, Proc. Centre for Math. Analysis, Australian National University.

Waksman, P. (1985) Plane polygons and a conjecture of Blaschke's. Adv. Appl. Prob. 17, 774-793.

Waksman, P. (1987) A stereological metric for plane domains. Adv. Appl. Math. 8, 38-52. 